\title{
Spatial Nonhomogeneous Poisson Process in Corrosion Management
}

\author{
J. López De La Cruz, ${ }^{a}$ S. P. Kuniewski, ${ }^{\text {b }}$ J. M. Van Noortwijk, ${ }^{\text {b,c }}$ and \\ M. A. Gutiérrez ${ }^{\mathrm{a}, \mathrm{d}, \mathrm{z}}$
}

${ }^{a}$ Faculty of Aerospace Engineering, ${ }^{b}$ Faculty of Electrical Engineering, Mathematics and Computer

Science, and ${ }^{d}$ Faculty of Mechanical, Maritime and Materials Engineering, Delft University of Technology,

The Netherlands

${ }^{c} H K V$ Consultants, Lelystad, The Netherlands

\begin{abstract}
A method to test the assumption of nonhomogeneous Poisson point processes is implemented to analyze corrosion pit patterns. The method is calibrated with three artificially generated patterns and manages to accurately assess whether a pattern distribution is random, regular, or clustered. The interevent and the nearest-neighbor statistics are employed to check the method's performance. Three empirical corrosion patterns are studied. The outcome of this investigation suggests that maximum pit depths are generally encountered where pit clusters are detected. This result is in agreement with previous studies. (C) 2008 The Electrochemical Society. [DOI: 10.1149/1.2926543] All rights reserved.
\end{abstract}

Manuscript submitted October 15, 2007; revised manuscript received April 11, 2008. Available electronically June 4, 2008.

One of the most common causes for structural and equipment failure in engineering is due to corrosion. In the United States, $\sim 3 \%$ of the gross national product is invested in corrosion management. ${ }^{1}$ In the last decade, significant progress has been made on the development of stochastic models that can be used to understand and characterize the corrosion process. The work of Shibata $^{2}$ and Melchers ${ }^{3}$ is worth mentioning.

Research has been mostly focused on developing time-dependent models. Spatial models started their incursion in the corrosion field a few years ago. Budiansky et al. ${ }^{4}$ applied the methods of Ripley to predict interactions among pitting sites. Ripley's methods are widely used in corrosion statistics. However, they introduce bias to the analysis when pits are widely separated. ${ }^{5}$ Punckt et al. ${ }^{6}$ analyzed the distribution of pits implementing the nearest-neighbor method. Organ et al. ${ }^{7}$ utilized the $\hat{L}$ estimator function to detect deviation from randomness in spatial pit patterns. LópezDeLaCruz et al. ${ }^{8}$ studied the interaction of pits in published empirical data applying the interevent distances estimator method. These methods assume that the process is stationary, which means that a constant number of pits is observed per unit area. Conversely, in a nonstationary process the number of pits per unit area becomes a function of the spatial location. The latter better represents what is encountered in practice.

In corrosion research, the spatial location of pits plays a fundamental role when maintenance models (preventive or corrective) are formulated. Insightful statistical models for maximum pit depth have been widely developed. ${ }^{9,10}$ Scarf and Laycock ${ }^{11}$ state that larger pitting events are found next to smaller neighbors whose growth is suppressed by the former. Consequently, maintenance models should be mostly focused where clusters of pits are present, because in these spots the risk of failure is higher.

In this paper, a statistical method to assess pit pattern distribution is implemented. The method, developed by Brix et al., ${ }^{12}$ has the advantage that it can be applied to different domain shapes and is very powerful detecting random, regular, or clustered patterns. Specifically, it can be applied to regular and irregular domains large enough to support cell division. The method is an extension of the classical complete spatial randomness test to nonstationary Poisson point processes. A pattern distribution is stationary when the distribution of points does not change toward any direction in the space. Thus, a stationary pattern is a pattern that exhibits regularity. In the corrosion field, the observed pitting events are frequently nonstationary and pit clusters are usually perceivable.

The method developed by Brix et al. ${ }^{12}$ is presented in the next section. The method is later validated with three artificially generated patterns, which are selected in such a way that the performance

${ }^{\text {z }}$ E-mail: m.a.gutierrez@tudelft.nl of the method is evaluated for random, regular, and clustered patterns, respectively. A subsequent section presents the application of the method to empirical corrosion data.

\section{Goodness of Fit of Spatially Nonhomogeneous Poisson Process}

Essentially, this method tests the nonhomogeneous Poisson point process (NH-PPP) assumption by performing an optimization routine. The intensity function, defined as the mean number of pits per unit area, is used here to find the optimum domain partition. The method is based on three main steps. In the first step, the domain is divided into cells scaled until the intensity function appears to be constant such that the same number of pits is observed in each cell. Then, cells containing at least two points are selected and the distribution of the distances among points per cell is computed. Finally, a statistical test is performed to check the nonhomogeneous Poisson assumption.

Intensity function and joint density distribution.- Frequently, the intensity function of the point processes encountered in practice is unknown. Therefore, the first step in the analysis is computing the intensity function, which is defined as the ratio between the number of events (corrosion pits) per cell and the cell size.

Consider the nonstationary Poisson point process $\Xi$ with twice differentiable intensity function $\lambda$. Let $x_{i}=\left\{\zeta_{i}, \eta_{i}\right\}$ stand for the spatial coordinates of one pit. Then, $\Xi=x_{1}, \ldots, x_{n}$ is the process composed by the spatial coordinates of $n$ pits in the observation window $W$, where $W$ is partitioned into $M$ cells $\mathcal{L}_{\delta}$ of size $\delta^{2}$. Figure 1 illustrates the process.

Applying Taylor's expansion to the intensity function evaluated at the middle of a cell $\mathcal{L}_{\delta}$

$$
\lambda(\mathbf{x})=\lambda\left\{1+\mathbf{x}^{T} \mathbf{a}+\mathbf{x}^{T} \mathbf{B} \mathbf{x}+\cdots\right\}
$$

where $\mathbf{x}$ is a vector containing the points in a cell, $\mathbf{a}$ is the gradient, and $\mathbf{B}$ is the matrix of second derivatives of $\lambda(x)$ or Hessian matrix. The dots at the end of the equation indicate a second-order expansion.

The density of any point of the process $\Xi$ lying in a cell $\mathcal{L}_{\S}$ conditioned on the number of points in that cell is $p(\mathbf{x})=\lambda(\mathbf{x}) / \Lambda_{\delta},{ }^{13}$
where $\Lambda_{\delta}=\int_{\mathcal{L}_{\delta}} \lambda(\mathbf{x}) d \mathbf{x}$. Then, the probability of observing a single point in $\mathcal{L}_{\delta}$ is given by

$$
p(\mathbf{x})=\frac{1}{\delta^{2}}\left\{1+\mathbf{x}^{T} \mathbf{a}+\mathbf{x}^{T} \mathbf{B} \mathbf{x}-\frac{\delta^{2}}{12} \operatorname{tr}(\mathbf{B})+\cdots\right\}
$$

where $\operatorname{tr}(\mathbf{B})$ is the trace of matrix $\mathbf{B} .^{12}$ In the same way, the probability of observing $n$ points in $\mathcal{L}_{\delta}$ is 


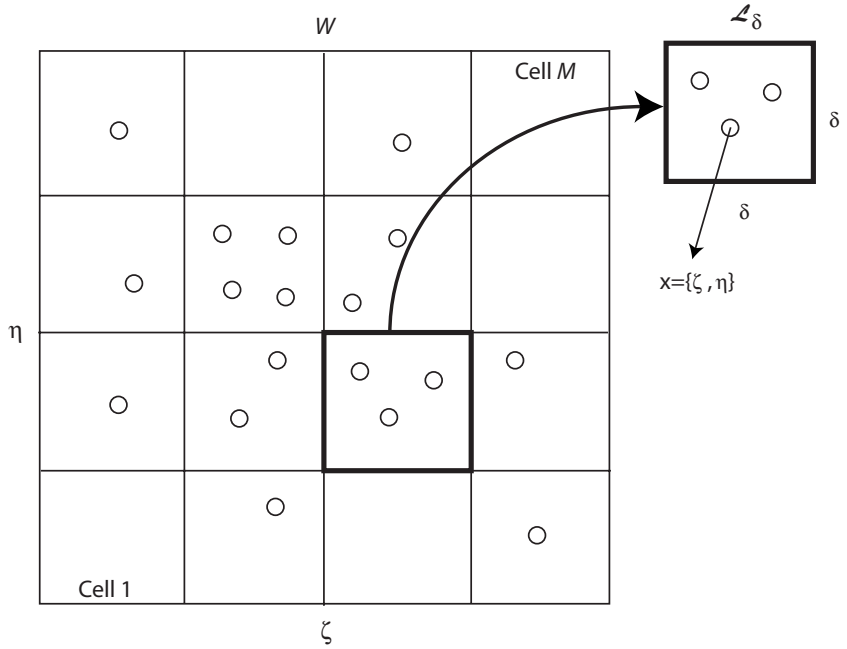

Figure 1. Schematic representation of the process $\Xi$ in the observation window $W$.

$$
\begin{aligned}
p\left(X_{1}, \ldots, X_{n}\right)= & \prod_{i=1}^{n} p\left(\mathbf{x}_{i}\right) \\
= & \frac{1}{\delta^{2 n}}\left[1+\left(\sum_{i=1}^{n} \mathbf{x}_{i}\right)^{T} \mathbf{a}+\sum_{i<j} \mathbf{x}_{i}^{T}\left(\mathbf{a a}^{T}\right) \mathbf{x}_{j}\right. \\
& \left.+\sum_{i=1}^{n} \mathbf{x}_{i}^{T} \mathbf{B} \mathbf{x}_{i}-n \frac{\delta^{2}}{12} \operatorname{tr}(\mathbf{B})+\cdots\right]
\end{aligned}
$$

Distribution of pits in a cell.- In order to analyze the point pattern contained in $\mathcal{L}_{\delta}$, two distance-based statistics $T_{\delta}$ are used: interevent and nearest neighbor. Generally, it is always recommended to analyze patterns with several methods. ${ }^{14}$ The point-to-event statistics is not applied here because it has been proven to perform poorly. $^{12}$

The distance-based statistics tests are used to compute the average distance among points in $\mathcal{L}_{\delta}$. If the intensity $\lambda$ is assumed to be constant, then the value of $\mathbf{a}$ and $\mathbf{B}$ is zero. In this way, the estimator for the $U$ value in each cell is approximated as $\hat{U}_{\delta}^{(n)}=\left|D\left(T_{\delta}^{(n)}\right)\right|$, where the $U$ value is defined as a realization of the random variable average distance among points in a cell, taken from its cumulative distribution function. Then, $\left|D\left(T_{\delta}^{(n)}\right)\right|$ is the set composed by the points that are separated a distance less than or equal to the average distance obtained by the distance-based statistics.

Taking into account the fact that the distance-based statistics are symmetric, Brix et al. ${ }^{12}$ developed a formula for $U_{\delta}^{(n)}$

$$
\begin{aligned}
U_{\delta}^{(n)}= & \hat{U}_{\delta}^{(n)}\left\{1-n \frac{\delta^{2}}{12} \operatorname{tr}(\mathbf{B})+\cdots\right\} \\
& +n \delta^{2} \int_{D\left(T_{\delta}^{(n)}\right)}\left(\mathbf{x}^{T} \mathbf{B} \mathbf{x}+\frac{n-1}{2} \mathbf{x}^{T} \mathbf{a a}^{T} \mathbf{x}\right) d \mathbf{x}
\end{aligned}
$$

When the statistics tests are carried out in more than one cell, for example, an observation window divided in $m$ cells of side length $\delta$, the results of the $\mathcal{L}_{m, \delta}$ cells are a distribution of $m U$ values.

Global test.-After the local statistical test (interevent or nearest-neighbor) is performed in each $\mathcal{L}_{m, \delta}$ and $U$ values $\hat{U}_{m, \delta}$ are found, it is possible to test whether the pattern follows a nonhomogeneous Poisson process; that is, the pattern exhibits a random behavior that cannot be considered as a stationary process.

The nonstationary Poisson assumption is checked performing a Kolmogorov-Smirnov (K-S) test. ${ }^{15}$ This test is used to decide if a sample comes from a population with a specific distribution. In this case, the hypothesis to test is whether the $U$ values $\left(U_{1}, \ldots, U_{m}\right)$ are independent and uniformly distributed between 0 and 1 . Thus, if the $U$ values are not uniformly distributed over $[0,1]$, then the nonstationary Poisson hypothesis is rejected and pit clustering is considered.

Optimum cell size.- The computed local $U$ value $U_{m, \delta}$ depends on the intensity of points per cell. Then, if the cell $\delta$ is large the approximation previously explained becomes poor and introduces bias, whereas for small $\delta$ the cells $\mathcal{L}_{m, \delta}$ contain few points, or no point at all, and the power of the test drops dramatically. ${ }^{12}$ Proofs and mathematical handling are not explained here. The reader can refer to Ref. 12 for further insights of the method.

In order to find the optimum cell size, another global test statistic is employed. The idea behind the cell optimization is to minimize both the asymptotic variance and the bias of the global test statistic. Equation 2 shows the weighted mean $\hat{H}$ of the local $\hat{U}$ values used as a global test. The weights $w_{m}=1_{n_{m} \geq 2}$ reflect that only cells containing at least two points are informative

$$
\hat{H}=\frac{\sum_{m \leq M} w_{m} \hat{U}_{m}}{\sum_{m \leq M} w_{m}}
$$

where $M$ is the total number of cells in the observation window. In each cell $\mathcal{L}_{m, \delta}$, the following approximation of Eq. 1 holds when $\Xi\left(\mathcal{L}_{m, \delta}\right)=n_{m}$

$$
\hat{U}_{m, \delta}=U_{m, \delta}+\delta^{2} \Theta_{m}+\cdots
$$

where

$$
\begin{aligned}
\Theta_{m}= & \frac{1}{12} n_{m} U_{m, \delta} \operatorname{tr}(\mathbf{B}) \\
& -\int_{D\left(T_{\delta}^{\left.n_{m}\right)}\right.}\left(n_{m} \mathbf{x}^{T} \mathbf{B}_{m} \mathbf{x}+\frac{n_{m}\left(n_{m}-1\right)}{2} \mathbf{x}^{T}\left(\mathbf{a}_{m} \mathbf{a}_{m}^{T}\right) \mathbf{x}\right) d \mathbf{x}
\end{aligned}
$$

After some mathematical manipulation, the biased asymptotic mean is computed as

$$
\hat{H} \rightarrow \mu=\frac{1}{2}+\delta^{2} E\left(\theta_{m} \mid n_{m} \geq 2\right)+\cdots
$$

where $E\left(\theta_{m} \mid n_{m} \geq 2\right)$ can be estimated as the mean of

$$
\begin{aligned}
& 1_{n_{m} \geq 2}\left\{\frac{n_{m}}{24} \operatorname{tr}\left(\mathbf{B}_{m}\right)-\int_{D\left(T_{\delta}\right)}\left[n_{m} \mathbf{x}^{T} \mathbf{B}_{m} \mathbf{x}\right.\right. \\
& \left.\left.+\frac{n_{m}\left(n_{m}-1\right)}{2} \mathbf{x}^{T}\left(\mathbf{a}_{m} \mathbf{a}_{m}^{T}\right) \mathbf{x}\right] d \mathbf{x}\right\}
\end{aligned}
$$

The integrals are approximated numerically by the Monte Carlo (MC) method, and the derivatives are estimated by finite differences. Alternatively, a quasi-Monte Carlo (QMC) method is implemented to check if both approximations are in agreement.

The asymptotic variance is computed with Eq. 6 . The term $\bar{w}$ is estimated as the ratio of cells containing at least two points

$$
\sigma^{2}=\frac{1}{12 \bar{w}}\{1+\cdots\}
$$

In the next section, the method is calibrated with three different patterns. In order to differentiate among them, a brief definition of each pattern is given. A random pattern (NH-PPP) is a pattern that does not show an obvious structure. In a regular pattern, the events are evenly distributed, whereas in a clustered pattern the events exhibit aggregation. ${ }^{13}$ If a pattern is found to be random or regularly distributed, then interaction among pits is discharged. ${ }^{8}$ Therefore, it is assumed that the presence of one pit neither influences the appearance of new pits nor enhances the growth of its neighboring pits. Conversely, if a pattern is found to be clustered, then pit interaction is plausible. 


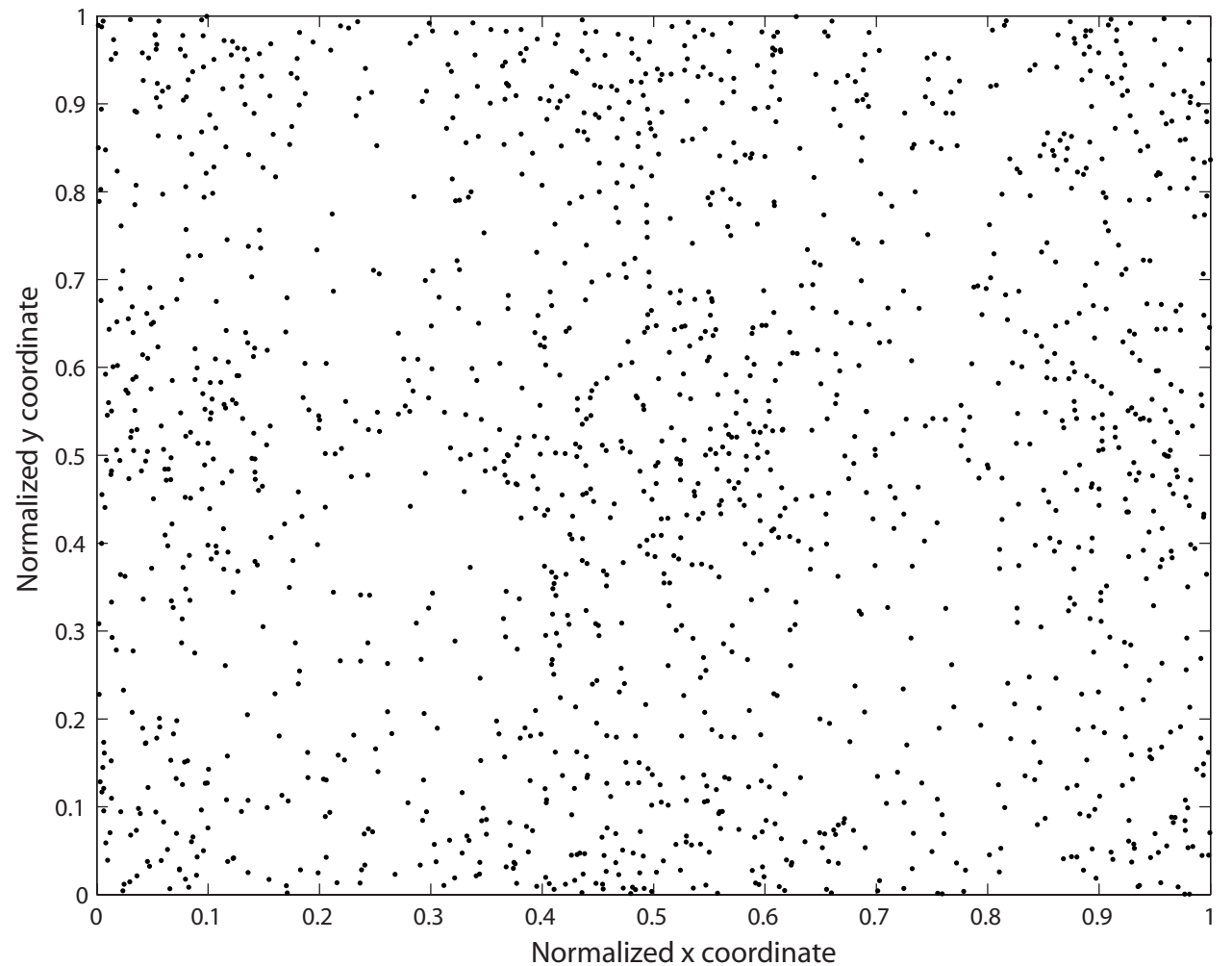

Figure 2. Random process generated with a NH-PPP.

\section{Method Validation}

The first pattern to analyze corresponds to NH-PPP generated from a Poisson process applying a thinning method. ${ }^{13}$ The process, illustrated in Fig. 2, contains 1514 points, and its intensity function is proportional to the function [2 $+\cos (0.4 \times \pi \times x)][2+\cos (0.4 \times \pi \times y)]$. The point pattern is generated in a window size $10 \times 10$ and further normalized to a window $1 \times 1$.

The first step in the analysis is to divide each side of the observation window into $n$ parts such that a constant intensity function can be expected in each cell. Because the pattern is normalized to a window size $1 \times 1$, the cell side $\delta$ is computed as the ratio between the observation window length (one in this case) divided by $n$. The intensity is then computed as the number of points contained in each cell divided by the cell area.

The gradient $\mathbf{a}$ and the Hessian $\mathbf{B}$ of the intensity function are required to compute the optimum cell size and the $U_{\text {values }}$ of each cell. Both terms are calculated by means of the finite differences method.

The next step is to compute the integration limits of Eq. 5. The set $D\left(T_{\delta}\right)$ is found with the help of the distance-based statistics. Thus, depending on the method one wants to implement, the average distance among points changes. Suppose that the interevent distance statistics is selected. Then, the average distance among points is computed from

$$
T^{\left(n_{m}\right)}=\frac{\sum_{i, j=1}^{n_{m}} T_{\mathrm{e} \mid \mathrm{e}}(i, j)}{2 n(n-1)}
$$

where $T_{\mathrm{e} \mid \mathrm{e}}(i, j)$ is the Euclidean distance and $n_{m}$ is the number of points in cell $m$.

Alternatively, if the nearest-neighbor statistics is chosen, then the average distance among points is computed from

$$
T^{\left(n_{m}\right)}=\frac{\sum_{i=1}^{n_{m}} T_{n n}(i)}{n}
$$

where $T_{n n}(i)=\inf \left\{d\left(x_{i}, x_{j}\right) ; 1 \leq j \leq n, j \neq i\right\}$.
From Eq. 7 and 8, a scalar value is always found. This value is denoted as $T_{\text {observed }}$ and used as an acceptance condition to give structure to the set $D\left(T_{\delta}\right)$. Thus, the integration set $D\left(T_{\delta}\right)$ is created with the $n_{m}$ uniform random samples that satisfy the condition $T^{n_{m}} \leq T_{\text {observed }}$.

The MC integration of Eq. 5 is performed evaluating the integrand $\left[n_{m} x_{1}^{T} \mathbf{B}_{m} x_{1}+\left\{\left[n_{m}\left(n_{m}-1\right)\right] / 2\right\} x_{1}^{T}\left(\mathbf{a}_{m} \mathbf{a}_{m}^{T}\right) x_{2}\right]$ in the first and second position of the sampled set $D\left(T_{\delta}\right)$. More specifically, the set $D\left(T_{\delta}\right)$ is composed by the coordinates $x-y$ of the points that satisfy $T^{n_{m}} \leq T_{\text {observed }}$. Thus, $x_{1}$ represents the $x-y$ coordinates in the first row of the matrix $D\left(T_{\delta}\right)$ and $x_{2}$ the coordinates in the second row. This is possible due to the permutation invariance assumption $T^{n_{m}}\left(x_{1}, \ldots, x_{n}\right)=T^{n_{m}}\left[x_{\sigma(1)}, \ldots, x_{\sigma(n)}\right]$. For the case at hand, the MC integration is performed with $N=5000$ samples.

In order to corroborate the results obtained with the MC method, a QMC algorithm is implemented. In the latter, the integration set $D\left(T_{\delta}\right)$ is constructed with all $N$ samples because they are restricted to fulfill the $T^{n_{m}} \leq T_{\text {observed }}$ condition.

From Eq. 4 , it is possible to observe that $\mu$ is minimized when its value is as close as possible to $\frac{1}{2}$ and for the asymptotic variance when its value is close to $\frac{1}{12}$. Thus, if $\delta^{2} E\left(\theta_{m} \mid n_{m} \geq 2\right)$ is not as close as possible to 0 and/or $\bar{w}$ is not close to 1 , then the amount of partitions $n$ of the observation window is increased (or decreased) until the results are the best estimates. Subsequently, when the optimum number of partitions has been found, the $U_{\text {values }}$ are computed from $\left|D\left(T_{\delta}^{(n)}\right)\right|$. This follows from the assumption that for sufficiently small cell sizes the intensity $\lambda(x)$ is constant.

A goodness-of-fit test is performed applying the $\mathrm{K}-\mathrm{S}$ method with a significance level of 0.05 . It is important to remark that, in the cases examined here, the null hypothesis is always assumed to be the NH-PPP.

For the pattern in Fig. 2, the routine optimization is performed starting from $n=5$, increasing each time by 1 until $n=35$. Table I presents the results obtained for the two distance-based statistics.

From Table I, it can be seen that the best estimates for 


\begin{tabular}{lcc}
\hline $\begin{array}{l}\text { Table I. NH-PPP simulation } \\
\text { nearest-neighbor statistics. }\end{array}$ & results for the interevent and \\
Variables & Interevent & Nearest-neighbor \\
\hline$\delta^{2} E\left(\theta_{m} \mid n_{m} \geq 2\right) \mathrm{MC}$ & 33.52 & 34.11 \\
$\delta^{2} E\left(\theta_{m} \mid n_{m} \geq 2\right) \mathrm{QMC}$ & 33.68 & 34.23 \\
$\bar{w}$ & 0.57 & 0.57 \\
Test $p_{\text {value }}$ & 0.749 & 0.830 \\
$n$ & 27 & 27 \\
$\delta$ & 0.037 & 0.037 \\
Hypothesis & Do not reject NH-PPP & Do not reject NH-PPP
\end{tabular}

$\delta^{2} E\left(\theta_{m} \mid n_{m} \geq 2\right)$ and $\bar{w}$ are found when the pattern is analyzed with $n^{2}=729$ cells. Moreover, Table I shows that the outcomes obtained by the two distance-based statistics for the hypothesis test are in perfect agreement. The NH-PPP hypothesis is not rejected.

Taking a closer look at the variables, it is possible to observe that the value of $\bar{w}$ is always the same for both methods. This is because $\bar{w}$ is measuring how informative the cells are and only depends on the cell size and the points contained in it. The results for the global test $p_{\text {values }}$ show that there is more confidence accepting a random distribution for the nearest-neighbor statistics.

Figure 3 presents the outcome of the $\mathrm{K}-\mathrm{S}$ test for the optimum $n \times n$ partition. The graph on the left illustrates the results obtained by the interevent statistics and the graph on the right by the nearestneighbor statistics. The diagonal line in both graphs represents the theoretical test results for a nonhomogeneous random pattern. The second line illustrates the results obtained.

In Fig. 3, it can be observed that the lines for the K-S test are very close to the theoretical line for both distance-based statistics. Therefore, it can be concluded that the pattern in Fig. 2 follows a NH-PPP and its distribution is random.

The pattern in Fig. 4 shows a Neyman-Scott point pattern. ${ }^{16}$ This process incorporates an explicit form of spatial clustering providing good basis for the modeling of aggregated spatial point patterns. The point pattern in Fig. 4 is generated with a Poisson parent process uniformly distributed on a unit square with intensity $\lambda=350$. The offspring process generates five uniformly distributed points on a square around a parent point that follows an exponential distribution with mean uniformly distributed on $[0,0.05]$. The pattern in Fig. 4 contains 1581 points.
In the same way as for the previous pattern, the optimum partition is sought. The computations are performed again in the interval $n=5$ until $n=35$, increasing in 1 partition each time. Hereafter, the whole process is repeated following the methodology described above.

Table II presents the results obtained for the pattern in Fig. 4. From Table II, it is possible to observe that the best estimates for $\delta^{2} E\left(\theta_{m} \mid n_{m} \geq 2\right)$ and $\bar{w}$ are obtained with 225 cells. In this case, the nearest-neighbor statistics perform better than the interevent statistics being that its estimates are closer to those expected. The $p_{\text {value }}$ for both statistics are very small, which leads to the rejection of the hypothesis of a random distribution in the pattern.

Figure 5 presents the results obtained after performing the K-S test for the optimum partition. Conversely, this time both lines depart significantly from the theoretical line. Furthermore, the location of the lines is below the theoretical one. Therefore, it can be concluded that the pattern presents clusters. The clusters size cannot be assessed by this method, but once a pattern is found to be clustered then a declustering process ${ }^{17}$ can be employed to analyze the clusters nature.

The previous examples demonstrate the power of the method when applied to random and clustered patterns. In the spatial statistics field, it is often the case that one method performs in a satisfactory way for aggregation but poorly when tested against a regular pattern. ${ }^{13}$ Figure 6 shows a regular pattern generated after substituting every grid joint by a point. The grid $15 \times 15$ is further distorted with a random displacement on $[0,0.05]$.

One more time, the optimization algorithm is carried out in the interval $n=5$ until $n=35$ increasing in 1 partition each time. The results obtained can be seen in Table III.

The results in Table III show that both statistics are in good concordance with their results. The best estimates are achieved with 121 cells, which is almost half of the cells needed to analyze the clustered pattern and six times less cells than for the nonstationary random pattern. This outcome derives from the fact that the intensity function is almost constant in each cell, which implies that significant variations do not exist throughout the pattern. Additionally, the result of the hypothesis test leads to reject the nonstationary Poisson point process, which means that the pattern is not random distributed.

The results obtained after performing the K-S test for the optimum partition are illustrated in Fig. 7. Similarly, as with the clustered pattern, both lines depart significantly from the theoretical line.
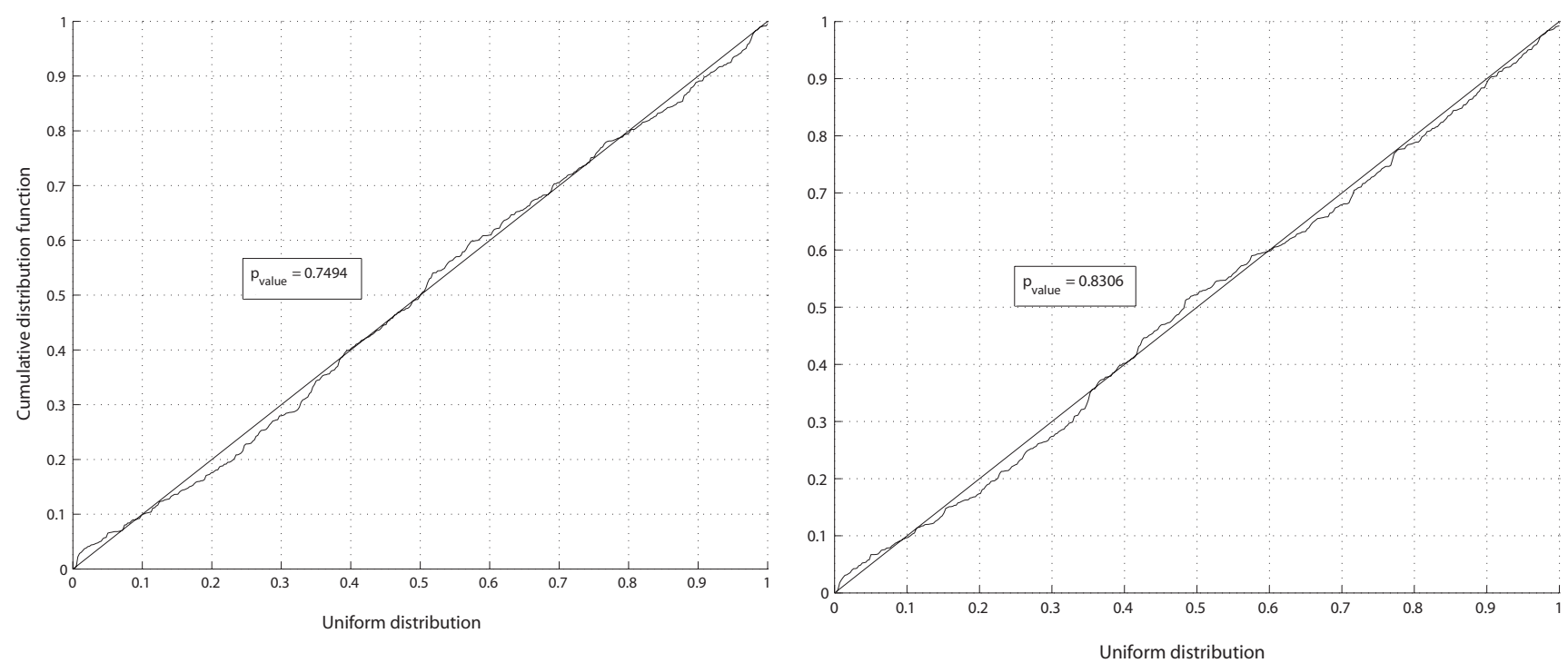

Figure 3. NH-PPP simulation results: (Left) Global test $p_{\text {value }}$ applying the interevent statistics and (right) global test $p_{\text {value }}$ applying the nearest-neighbor statistics. 


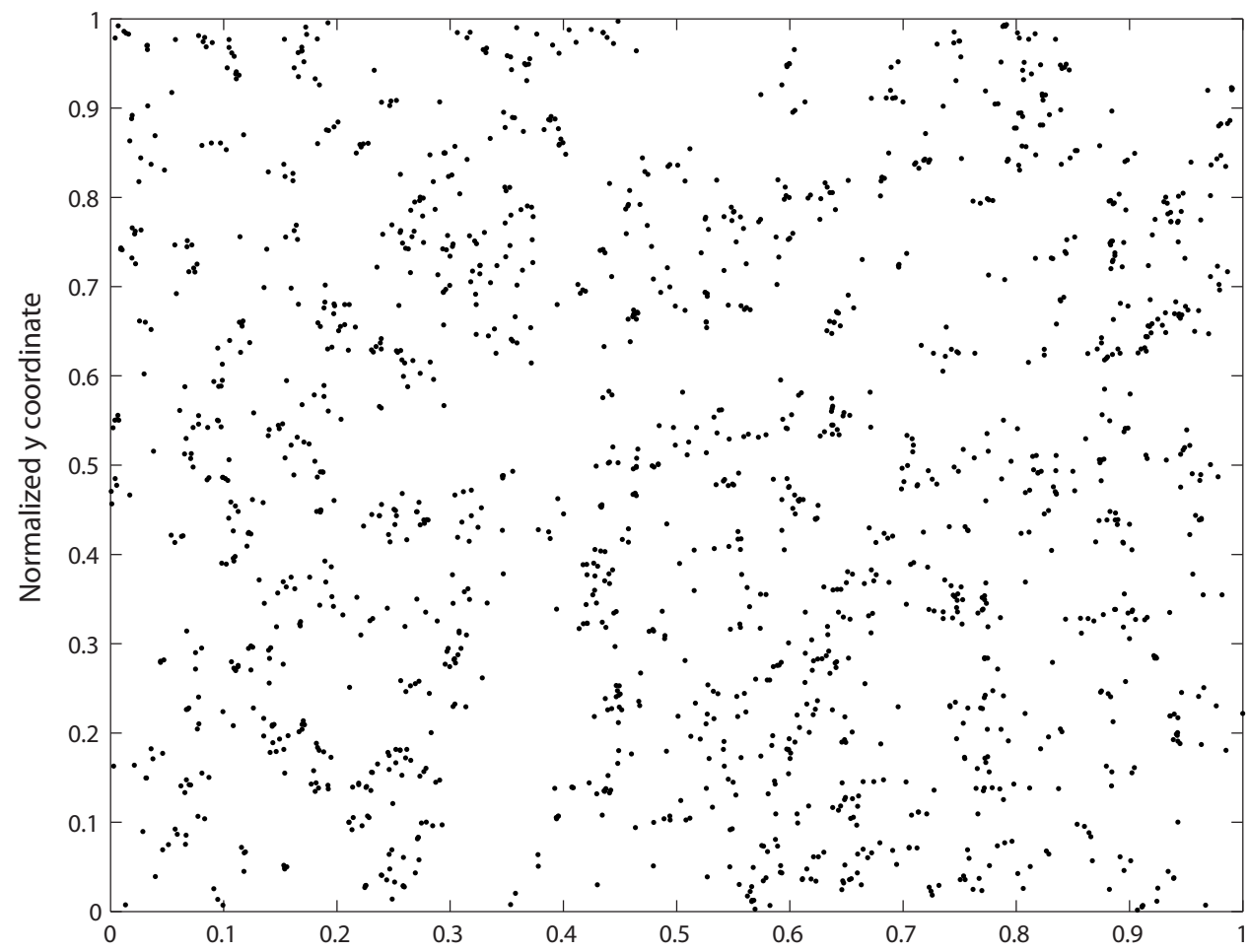

Figure 4. Cluster pattern generated with a Neyman-Scott process with five offsprings per parent.

\section{Normalized $x$ coordinate}

Table II. Neyman-Scott process simulation results for the interevent and nearest-neighbor statistics.

\begin{tabular}{lcc} 
Variables & Interevent & Nearest-neighbor \\
\hline$\delta^{2} E\left(\theta_{m} \mid n_{m} \geq 2\right) \mathrm{MC}$ & -3.67 & 0.25 \\
$\delta^{2} E\left(\theta_{m} \mid n_{m} \geq 2\right) \mathrm{QMC}$ & -3.28 & 1.25 \\
$\bar{w}$ & 0.87 & 0.87 \\
Test $p_{\text {value }}$ & $1.858 \times 10^{-24}$ & $4.130 \times 10^{-38}$ \\
$n$ & 15 & 15 \\
$\delta$ & 0.066 & 0.066 \\
Hypothesis & Reject NH-PPP & Reject NH-PPP
\end{tabular}

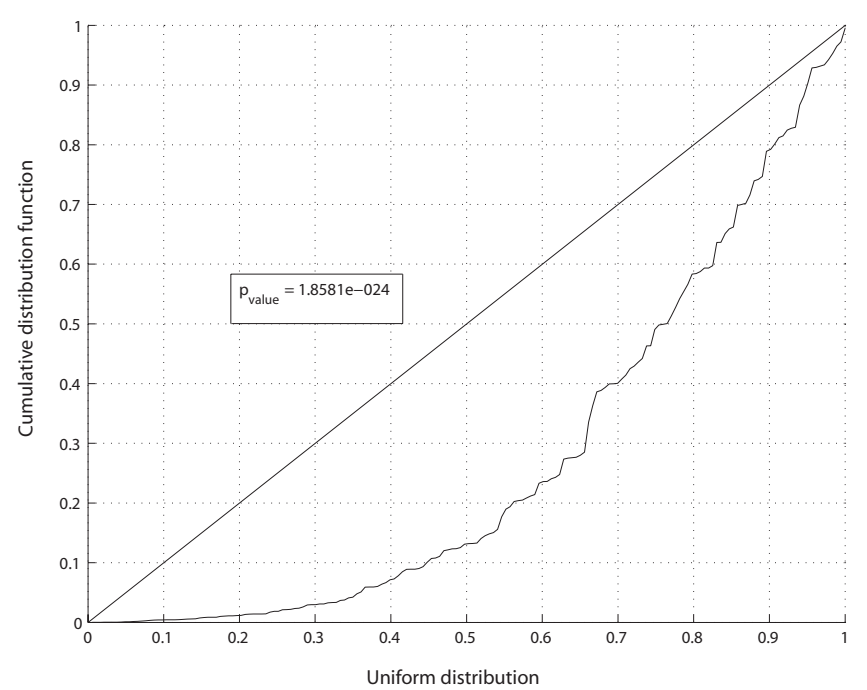

Furthermore, it can be seen that the lines are located above the theoretical one. Therefore, it can be concluded that the pattern presents regularity.

\section{Empirical Data Analysis}

In general, most maintenance models are focused on the study of mechanisms that lead to maximum pit depth and, therefore, to failure. ${ }^{18}$ The elapsed time until the maximum pit depth is observed is, in most cases, the variable of interest. Similarly, more attention should be given to the spatial analysis of pits because the location of deep pits is also significant in practice. The spatial distribution of pits helps one to understand the mechanism that drives pits to grow

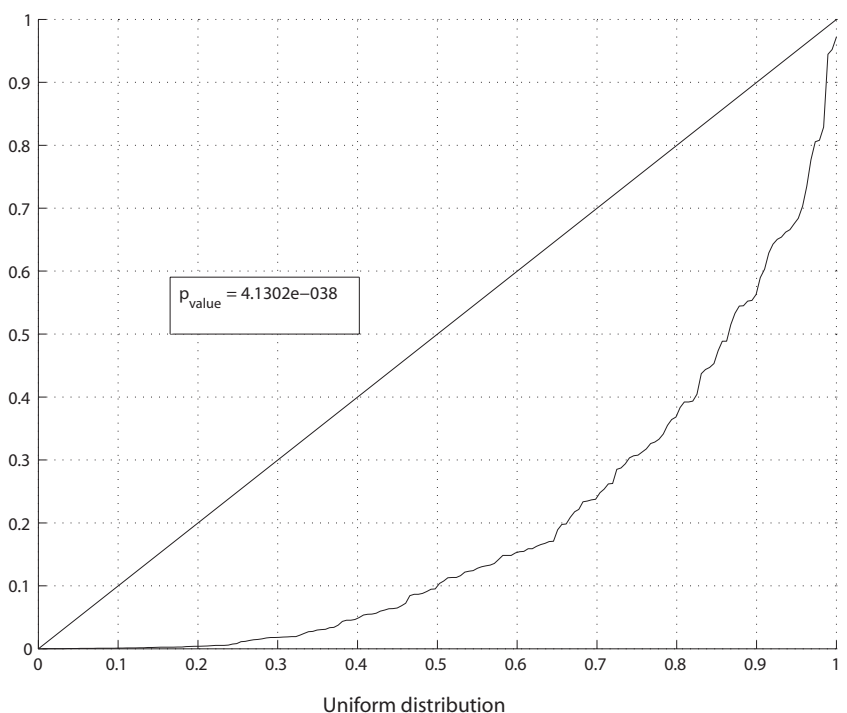

Figure 5. Neyman-Scott process simulation results: (Left) Global test $p_{\text {value }}$ applying the interevent statistics and (right) global test $p_{\text {value }}$ applying the nearest-neighbor statistics. 


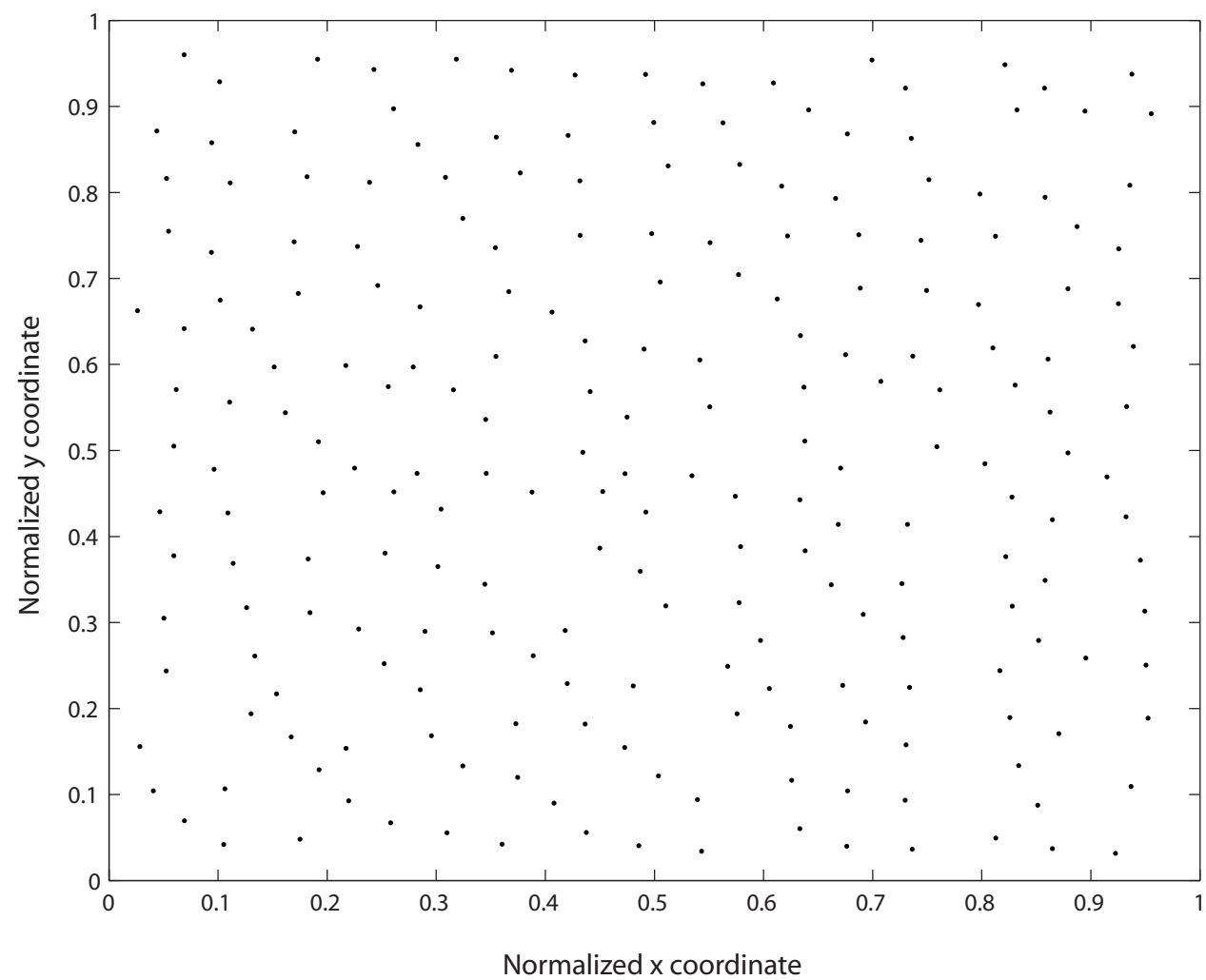

Figure 6. Regular pattern computed in a distorted rectangular grid.

\begin{tabular}{lcc}
\hline \multicolumn{2}{l}{$\begin{array}{l}\text { Table III. Regular process simulation results for the interevent } \\
\text { and nearest-neighbor statistics. }\end{array}$} \\
Variables & Interevent & Nearest-neighbor \\
\hline$\delta^{2} E\left(\theta_{m} \mid n_{m} \geq 2\right) \mathrm{MC}$ & 0.89 & 0.89 \\
$\delta^{2} E\left(\theta_{m} \mid n_{m} \geq 2\right) \mathrm{QMC}$ & 0.92 & 0.91 \\
$\bar{w}$ & 0.65 & 0.65 \\
Test $p_{\text {value }}$ & $4.806 \times 10^{-15}$ & $2.718 \times 10^{-14}$ \\
$n$ & 11 & 11 \\
$\delta$ & 0.090 & 0.090 \\
Hypothesis & Reject NH-PPP & Reject NH-PPP
\end{tabular}

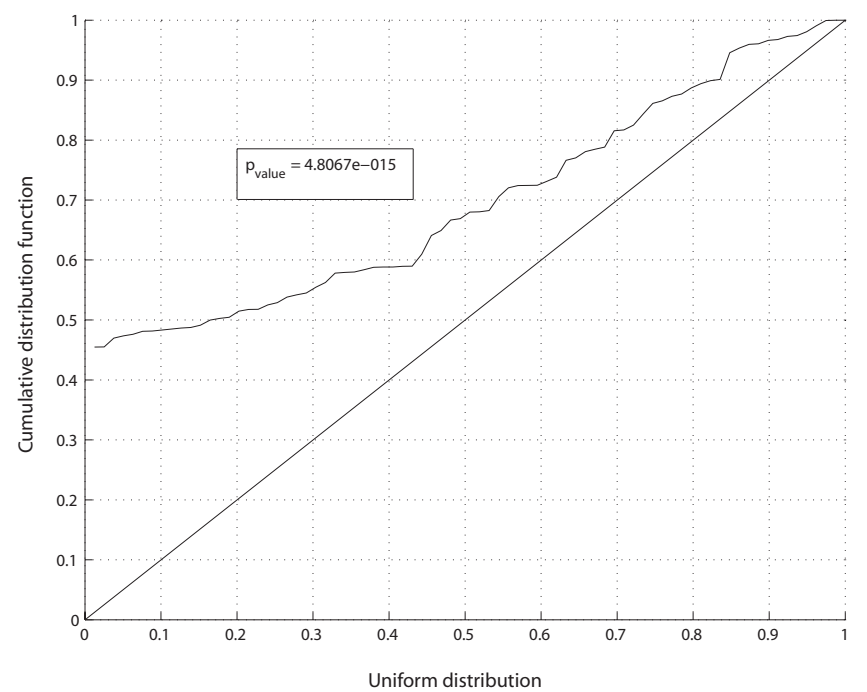

or passivate. Electrochemical studies suggest that neighboring pits interact in such a way that the growth of one pit inhibits the development of others. ${ }^{11}$ By means of spatial statistics, pit neighborhoods are detected at early stages; thus, corrective measures can be applied to prevent further damage to a structure.

Pit depths of neighboring pits are believed to be dependent, and the most prominent pit is often selected as representative of the pit cluster. $^{11,19}$ Performing the spatial analysis proposed here, it is possible to determine whether the pattern under analysis presents clustered, regular, or random pit distribution. If a random distribution of pits is found, then the pit depths are assumed to be independent. Consequently, standard statistical methods, such as the maximum

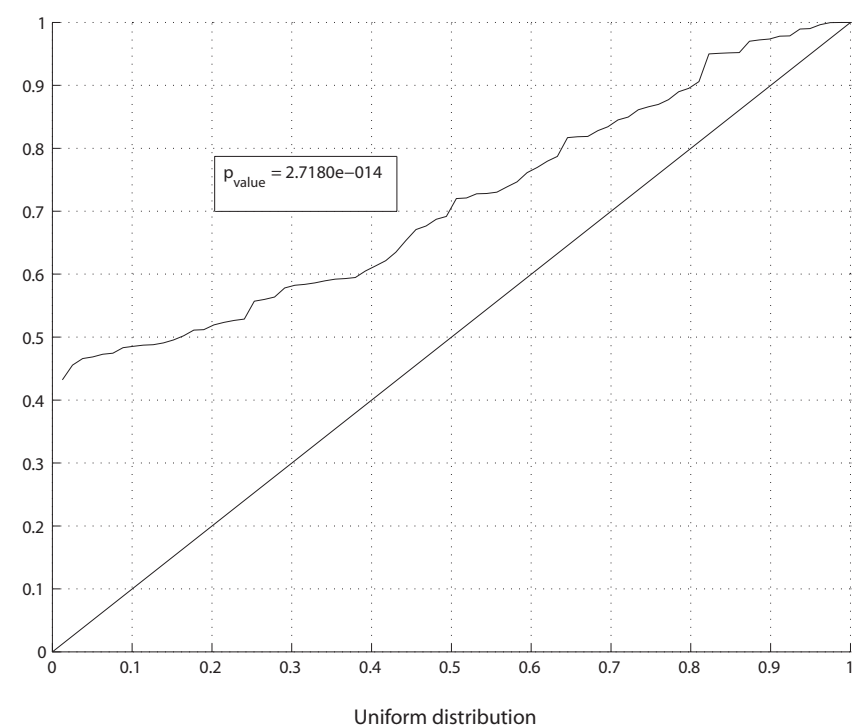

Figure 7. Regular process simulation results: (Left) Global test $p_{\text {value }}$ applying the interevent statistics and (right) global test $p_{\text {value }}$ applying the nearest-neighbor statistics. 


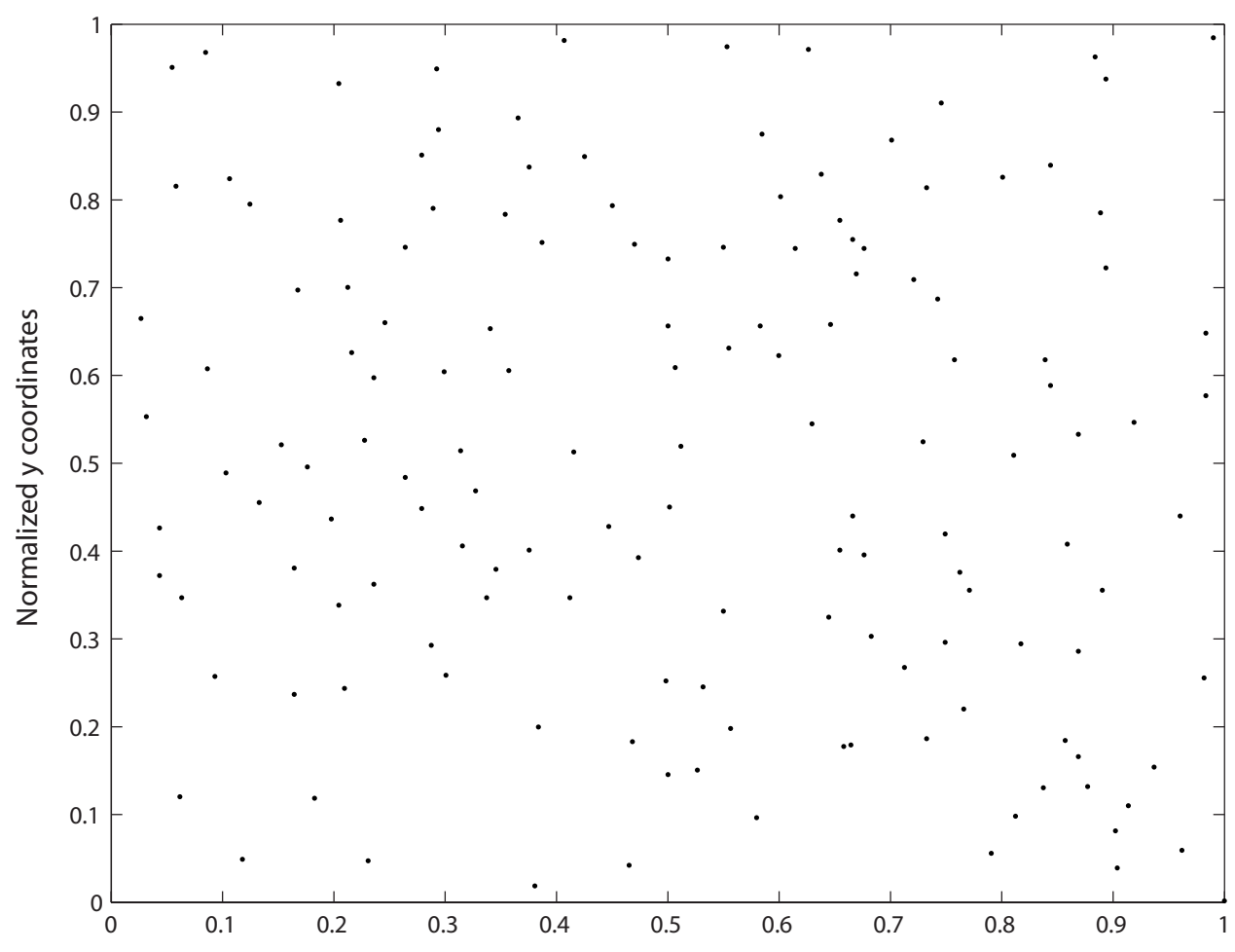

Figure 8. 2024-T3 aluminum alloy after exposure to $0.5 \mathrm{M} \mathrm{NaCl}$ solution for $72 \mathrm{~h}$ at $40^{\circ} \mathrm{C}$.

Normalized $x$ coordinates

likelihood estimator, can be used to get insight into the statistical properties. Conversely, if a clustered pattern is found, then the pit depths are no longer independent and special statistics, such as extreme value analysis and declustering methods, should be applied. ${ }^{17}$

Three different pit patterns encountered in practice are studied. The pattern in Fig. $8^{20}$ is used to illustrate the power of the method when a regular empirical pattern is observed. The pattern corresponds to an aluminum alloy 2024-T3 after exposure to $0.5 \mathrm{M} \mathrm{NaCl}$ solution for $72 \mathrm{~h}$ at $40^{\circ} \mathrm{C}$. In their paper, ${ }^{20}$ the authors implemented diverse spatial statistics techniques in their quest to find the spatial distribution of pits. They based the pattern analysis on the location of pit centroids, and other physical variables are assumed to be implicit in the modeling. Finally, they concluded that pits tend to be regularly spaced but further analysis is needed.

The optimization procedure for this case is performed for partitions between $n=5$ to 20, increasing in one partition each time. In Table IV, the results of the analysis are presented. The best estimates are found with 81 cells.

Table IV. 2024-T3 aluminum alloy pattern simulation results for
the interevent and nearest-neighbor statistics.

\begin{tabular}{lcc} 
Variables & Interevent & Nearest-neighbor \\
\hline$\delta^{2} E\left(\theta_{m} \mid n_{m} \geq 2\right) \mathrm{MC}$ & -2.70 & -2.65 \\
$\delta^{2} E\left(\theta_{m} \mid n_{m} \geq 2\right) \mathrm{QMC}$ & -2.61 & -2.62 \\
$\bar{w}$ & 0.54 & 0.54 \\
Test $p_{\text {value }}$ & 0.086 & 0.030 \\
$n$ & 9 & 9 \\
$\delta$ & 0.11 & 0.11 \\
Hypothesis & Do not reject NH-PPP & Reject NH-PPP
\end{tabular}

This time the statistics methods do not coincide in their results. The interevent statistics do not reject the NH-PPP hypothesis, whereas the nearest-neighbor statistics do reject it. However, for both statistics, the test $p_{\text {value }}$ is very close to the rejection limit. In Fig. 9, the K-S test results are illustrated. In both graphs, it can be seen that the lines are located above the theoretical line. The K-S test result line for interevent statistics crosses the theoretical line twice but in proportion is mostly above it. In evidence of the results and with the aid of the graphs in Fig. 9, it is possible to conclude that the pattern in Fig. 8 presents regularity.

Figure 10 shows the pit depth distribution at the bottom of a pipeline section used in oil transport. In Fig. 10, it is possible to observe that the deepest pits are located toward the bottom part of the pipeline. The positive values in the pit depths represent pit penetration in the metal. The negative values are due to passivated pits covered by a rust layer. The data are taken with an ultrasonic measuring device that determines pit depths. The ultrasonic measuring method is based on the time-of-flight diffraction technique, introduced in $1977 .^{21}$ This method has been tested in pipelines delivering significant results. ${ }^{22}$ The measurements performed by the system have a standard deviation or error of $1 \mathrm{~mm}$, and the pipe wall thickness is equal to $19 \mathrm{~mm}$. In order to obtain the collection of data, the device is moved every $5 \mathrm{~mm}$ in the radial direction inside the pipeline. The top of the pipeline is not examined because it does not exhibit damage. When the device has finished its radial trajectory, then it is moved $30 \mathrm{~mm}$ in the axial direction and the process is repeated. Figure 11 illustrates the process. The resolution of the method has proven to be enough to detect corrosion defects inside a pipeline. Nevertheless, its empirical accuracy is not enough to detect pits in a microscopic scale. Therefore, a cluster pattern composed of many of these microscopic pits can be overlooked as random in a macroscale.

Figures 12 and 13 present the pit pattern distributions of the pipeline for two different pit depth thresholds. In this paper, spatial analysis is used as a tool to find how max pit depths are distributed 

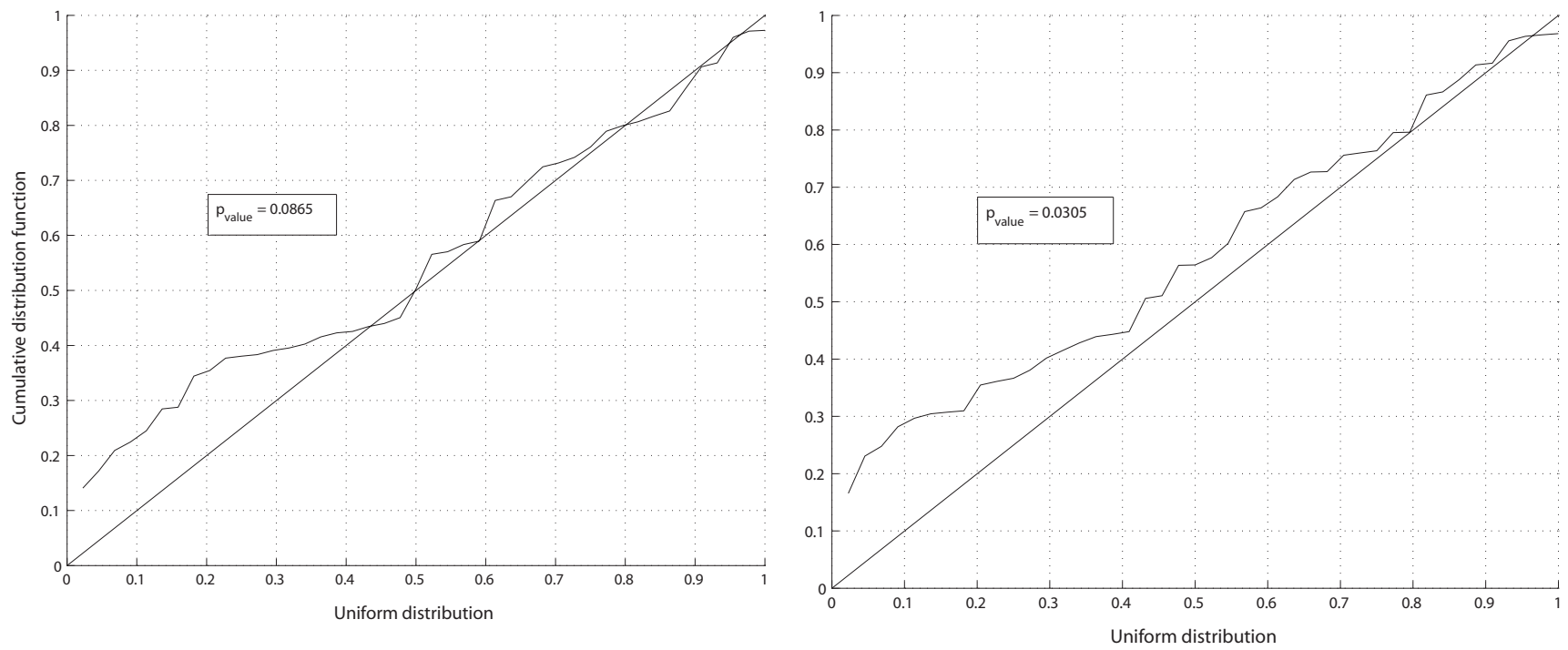

Figure 9. 2024-T3 aluminum alloy pattern simulation results: (Left) Global test $p_{\text {value }}$ applying the interevent statistics and (right) global test $p_{\text {value }}$ applying the nearest-neighbor statistics.

inside the pipe. Figure 12 presents the pitting events whose depth is $>0.5 \mathrm{~mm}$ (threshold). The data are normalized to facilitate analysis. The optimization routine is carried out once again for partitions between $n=5$ to 20 increasing in one partition each time. Table $\mathrm{V}$ presents the results obtained for the optimum partition.

From Table V, it can be seen that both statistics reject the NHPPP hypothesis with very small test $p_{\text {value }}$. The interevent statistics finds the best estimates when the observation window is divided in 169 cells, whereas the nearest-neighbor statistics, when the observation window is divided into 121 . For this pattern, it is possible to observe that the optimum partitions are found with high estimates for the biased asymptotic mean. These results are due to the marked irregularity of the pattern. Therefore, the computed values for Hessian and gradient, which describe the variation of the pattern in space, are also high.

Figure 13 shows the K-S test results for the optimum partitions in both statistics. Both lines deviate significantly from the theoretical line. Moreover, the lines are located below the theoretical line, which leads to the conclusion that the pattern exhibits clusters.

In Fig. 14, the same pipeline pattern is presented with a threshold of $0.69 \mathrm{~mm}$. That is, only pit depths of $>0.69 \mathrm{~mm}$ are taken into account. Table VI shows the results obtained after the optimization procedure is carried out. The process is performed in the same way as for the pattern in Fig. 12 .

From Table VI, it is observed that both statistics agree again in their result and reject the NH-PPP hypothesis with a small test $p_{\text {value }}$. The interevent statistics finds its best estimates for 100 cells, whereas the nearest-neighbor, for 49 .

Figure 15 illustrates the results obtained for the K-S test. One more time, both lines show a significant deviation from the theoretical line. In addition, the lines are located below the theoretical line in the same way as for the pattern in Fig. 12. These results lead to the conclusion that the pattern in Fig. 14 presents clusters.

Comparing the results obtained for the patterns in Fig. 12 and 14, it is possible to observe that, even after the pit depth threshold is increased (so that only deep pits are analyzed), the method delivers the same outcome. This result confirms that the maximum pit depths are found in clusters as previously proposed by other authors. ${ }^{1}$

In order to show the potential of this method in practice, it is important to keep in mind that the size of the surface to analyze does not represent an obstacle for this method. Actually, a bigger surface is advantageous because analysis at small scales can overlook clus-

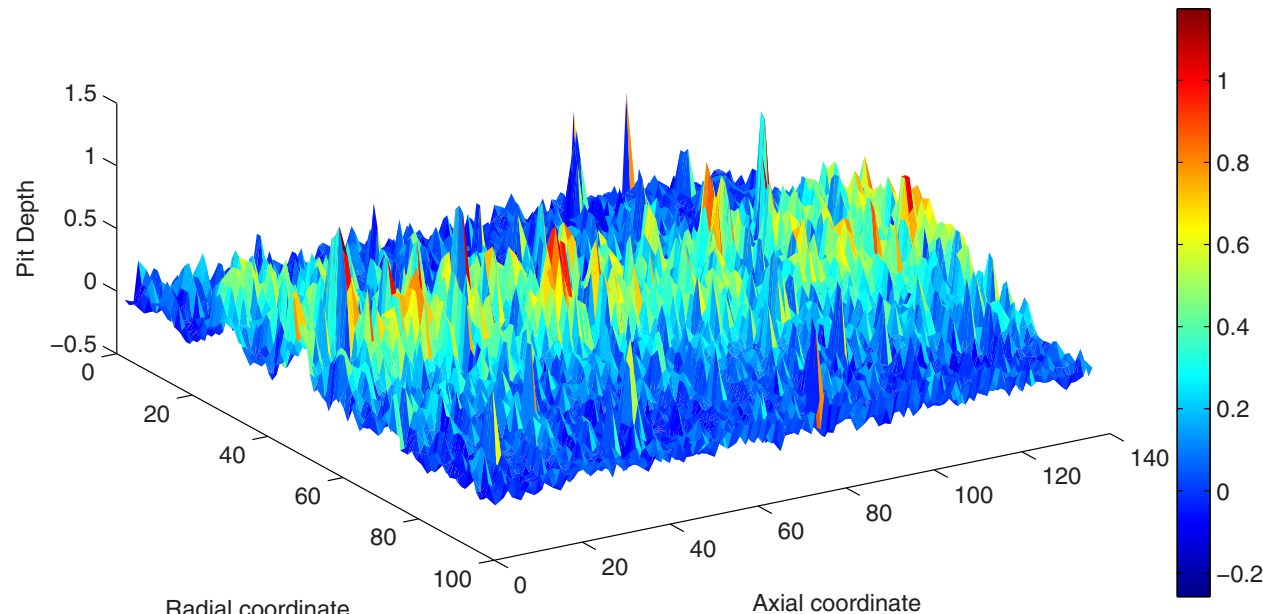

Figure 10. (Color online) Pit depth distribution at the bottom of a pipeline section. The positive values represent pit penetration in the metal. Negative values are due to passivated pits covered by a rust layer. 


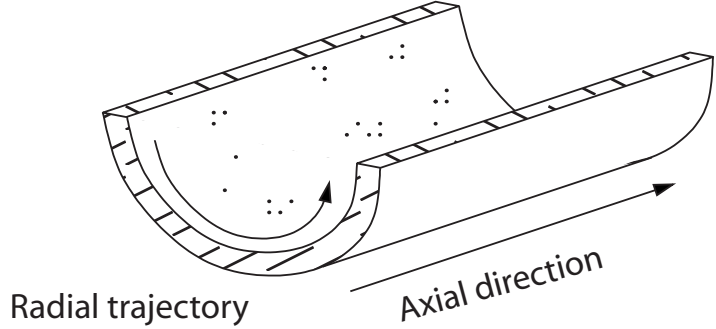

Figure 11. Schematic representation of the procedure to obtain pit depth measurements.

ter patterns identifying them as regular or random. Moreover, standard scale sizes cannot be estimated because cluster patterns are found at different scales. Consequently, the optimum scale size computed for a pattern where the pits are measured in a macroscale cannot provide optimum results when the same scale is brought into a microlevel. However, if the pits at a microlevel can be detected, then the analysis should be carried on with the inclusion of these pits as this will increase the method's performance.

Additionally, the time variable also plays a major role when analyzing corrosion samples. Thus, a pit pattern observed at time $t_{1}$ can differ from a pattern observed at time $t_{2}$ because some pits may have changed its state. For this reason, the inclusion of passive pits into the model is essential because their activation can be predicted by analyzing any change in the state of their neighboring pits.

\section{Conclusions}

Brix et al. ${ }^{12}$ proposed a method based on the complete spatial randomness test to assess the goodness of fit of a nonstationary Poisson spatial process. In this paper, this method is not only used to assess whether a pattern follows a NH-PPP but also to detect clustered or regular pit patterns and to find the optimum number of partitions that optimize the analysis. The advantage of this method

\begin{tabular}{lcc}
\hline \multicolumn{3}{l}{$\begin{array}{l}\text { Table V. Oil pipe pit pattern (threshold above } 0.5 \\
\text { mm) simulation } \\
\text { results for the interevent and nearest-neighbor statistics. }\end{array}$} \\
\begin{tabular}{lcc} 
Variables & Interevent & Nearest-neighbor \\
\hline$\delta^{2} E\left(\theta_{m} \mid n_{m} \geq 2\right) \mathrm{MC}$ & -239.36 & -731.14 \\
$\delta^{2} E\left(\theta_{m} \mid n_{m} \geq 2\right) \mathrm{QMC}$ & -233.85 & -727.56 \\
$\bar{w}$ & 0.39 & 0.44 \\
Test $p_{\text {value }}$ & $7.417 \times 10^{-18}$ & $2.211 \times 10^{-17}$ \\
$n$ & 13 & 11 \\
$\delta$ & 0.07 & 0.09 \\
Hypothesis & Reject NH-PPP & Reject NH-PPP
\end{tabular}
\end{tabular}

over the widely used Ripley's methods is the fact that bias is not introduced to the analysis when pits are widely separated. In Ripley's methods, the estimator becomes unbounded when the distance among pits increases. ${ }^{5,8}$

The method is applied to three artificially generated patterns, each one with specific characteristics (random, clustered, or regular) in order to calibrate the method. Furthermore, two distance-based statistics are used to compare the method performance. Both statistics behave similarly and proved to be efficient in the detection of random, clustered, and regular patterns.

Three empirical corrosion patterns are presented. These are analyzed implementing both spatial statistics. The first pattern, a 2024-T3 aluminum alloy, does not reject the NH-PPP but because the K-S test line is located above the theoretical line, it can be concluded that the pattern exhibits regularity. This result is in agreement with what the authors found ${ }^{20}$ applying several spatial statistics methods in the analysis of pit patterns.

The second and third patterns illustrate the distribution of pits in a pipeline for oil transport. These patterns are analyzed taking into account two different pit depth thresholds. For both thresholds, the NH-PPP hypotheses is rejected and the K-S test lines are located below the theoretical line. Hence, maximum pit depths are observed in places where clusters of pits are encountered. This result is in agreement with what other authors have proposed and with what the

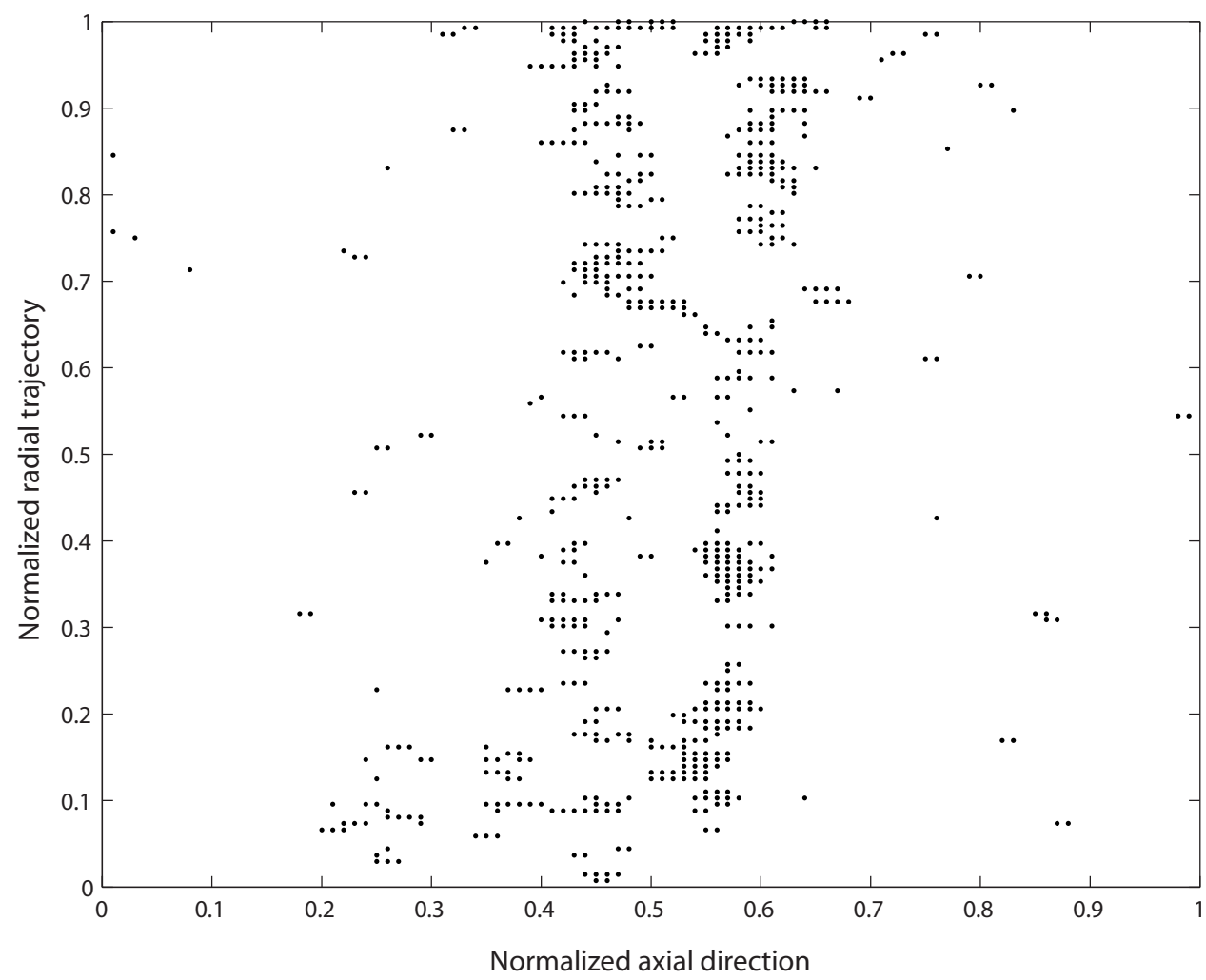

Figure 12. Oil pipe with pit depth threshold of $>0.5 \mathrm{~mm}$. 


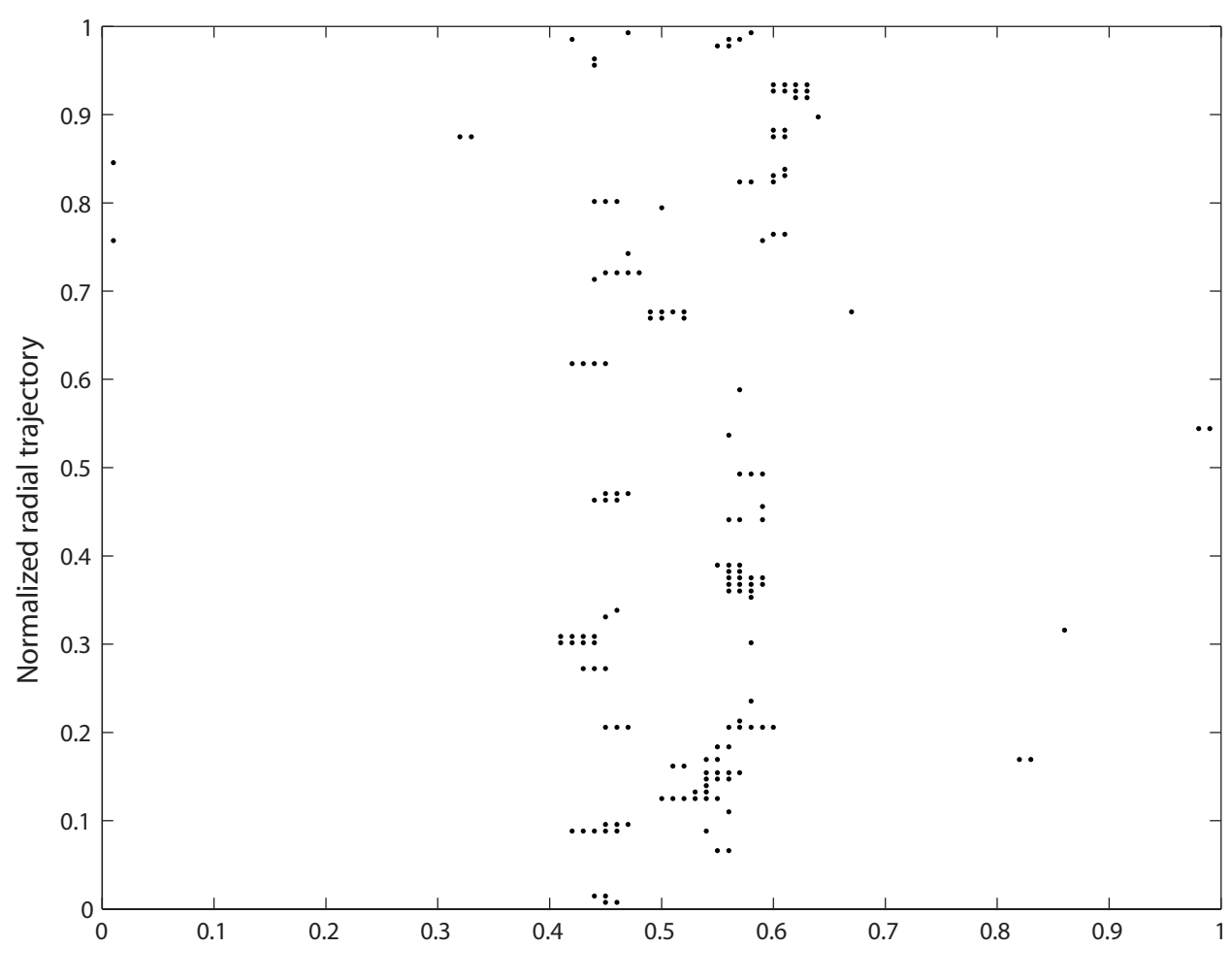

Figure 13. Oil pipe with pit depth threshold of $>0.69 \mathrm{~mm}$.

Normalized axial direction

\section{Table VI. Oil pipe pit pattern (threshold above $0.69 \mathrm{~mm}$ ) simu- lation results for the interevent and nearest-neighbor statistics.}

Variables

$\delta^{2} E\left(\theta_{m} \mid n_{m} \geq 2\right) \mathrm{MC}$

$\delta^{2} E\left(\theta_{m} \mid n_{m} \geq 2\right) \mathrm{QMC}$

Test $p_{\text {value }}$

$n$

Hypothesis
Interevent

$-64.42$

$-62.74$

0.24

$4.440 \times 10^{-7}$

10

0.09

Reject NH-PPP
Nearest-neighbor

$-124.19$

$-124.03$

0.36

$1.333 \times 10^{-7}$

7

0.14

Reject NH-PPP electrochemical theory suggests. ${ }^{11,19}$ Further experiments and empirical data are necessary in order to relate spatial patterns to a specific material, microstructure, or environmental conditions.

Applications of this method in practice could save significant effort and capital, because safeguarding can be focused in zones where clusters of pits are observed. Thus, inspection of large equipment, such as pipelines and offshore equipment, can be performed in a more efficient way via cluster identification.
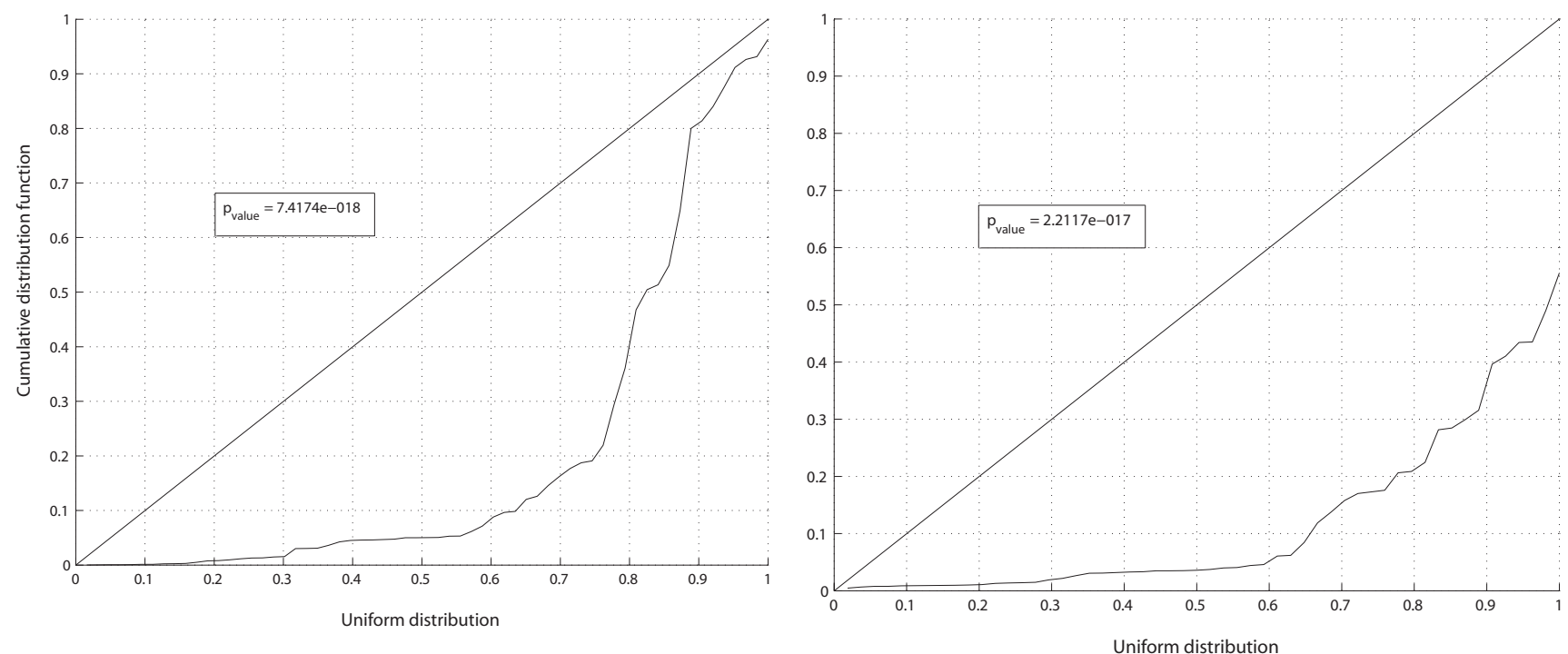

Figure 14. Oil pipe pit pattern (threshold of $>0.5 \mathrm{~mm}$ ) simulation results: (Left) Global test $p_{\text {value }}$ applying the interevent statistics and (right) global test $p_{\text {value }}$ applying the nearest-neighbor statistics. 

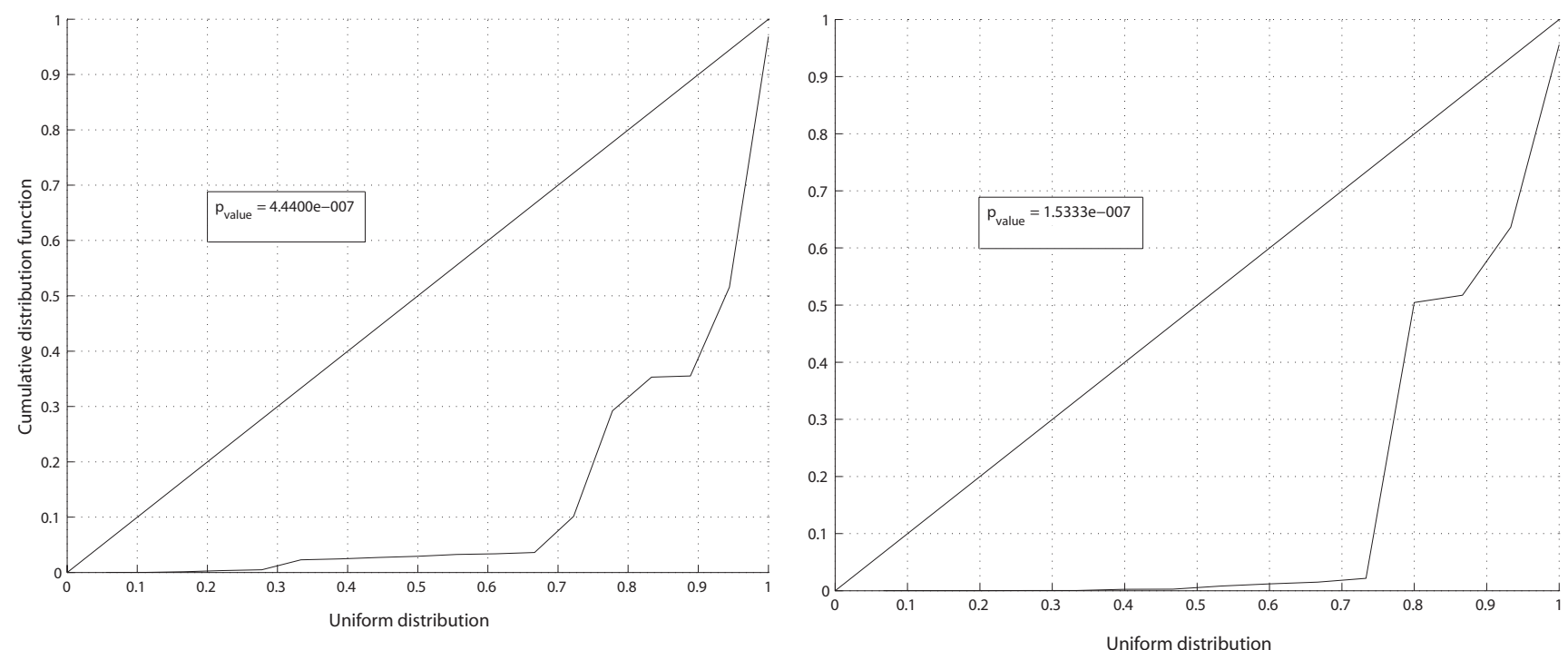

Figure 15. Oil pipe pit pattern (threshold of $>0.69 \mathrm{~mm}$ ) simulation results: (Left) Global test $p_{\text {value }}$ applying the interevent statistics and (right) global test $p_{\text {value }}$ applying the nearest-neighbor statistics.

\section{Acknowledgments}

This work is supported by the Technology Foundation STW, Applied Science division of NWO, and the technology programme of the Dutch Ministry of Economic Affairs. The authors thank Dr. J. A. M. van der Weide of Delft University of Technology and Dr. MarieColette van Lieshout of the Centrum voor Wiskunde en Informatica (CWI) for their help.

Delft University of Technology assisted in meeting the publication costs of this article.

\section{References}

1. R. L. Jones, Mat. Perf., 35, 763 (1996)

2. T. Shibata, Corrosion (Houston), 52, 813 (1996).

3. R. E. Melchers, Corros. Sci., 10, 2391 (2005).

4. N. D. Budiansky, L. Organ, J. L. Hudson, and J. R. Scully, J. Electrochem. Soc., 152, B152 (2005).

5. P. F. M. Dekking, C. Kraaikamp, H. P. Lopuhaa, and L. E. Meester, Probability and Statistics for the 21st Century, Delft University of Technology, Delft (2002).

6. C. Punckt, M. Bölscher, H. H. Rotermund, A. S. Mikhailov, L. Organ, N. Budiansky, J. R. Scully, and J. L. Hudson, Science, 305, 1133 (2004).

7. L. Organ, J. R. Scully, A. S. Mikhailov, and J. L. Hudson, Electrochim. Acta, 51,
$225(2005)$

8. J. LópezDeLaCruz, R. H. A. Lindelauf, L. Koene, and M. A. Gutiérrez, Electrochem. Commun., 9, 325 (2007)

9. R. E. Melchers, Corrosion (Houston), 60, 824 (2004).

10. R. E. Melchers, Corrosion (Houston), 60, 937 (2004).

11. P. A. Scarf and P. J. Laycock, Appl. Stat., 23, 621 (1996).

12. A. Brix, R. Senoussi, P. Couteron, and J. Chadoeuf, Biometrika, 88, 487 (2001).

13. P. J. Diggle, Statistical Analysis of Spatial Point Patterns, p. 46, Academic Press, New York (1983).

14. D. R. Cox, Scand. J. Stat., 4, 49 (1977).

15. I. M. Chakravarti, R. G. Laha, and J. Roy, Handbook of Methods of Applied Statistics, Vol. I, p. 392, John Wiley \& Sons, Hoboken, NJ (1967).

16. J. Neyman and E. L. Scott, J. R. Stat. Soc. Ser. B (Methodol.), B20, 1 (1958)

17. S. Coles, An Introduction to Statistical Modelling of Extreme Value Theory, p. 208 , Springer, London (2001)

18. S. P. Kuniewski and J. M. van Noortwijk, in European Safety and Reliability (ESREL), Terje Aven and Jan Erik Vinnem, Editors, Taylor and Francis Group, London (2007)

19. P. J. Laycock and P. A. Scarf, Corros. Sci., 35, 135 (1993).

20. N. R. Cawley and D. G. Harlow, J. Mater. Sci., 315127 (1996).

21. M. G. Silk, in Research Techniques in Non-destructive Testing, Vol. III, R. S. Sharpe, Editor, Academic Press, New York (1977).

22. M. Moles, N. Dube, S. Labbe, and E. Ginzel, J. Pressure Vessel Technol., 127, 35 (2005). 